\title{
Global attractivity for Volterra type Hadamard fractional integral equations in Fréchet spaces
}

https://doi.org/10.1515/dema-2018-0009

Received January 10, 2017; accepted April 9, 2018

\begin{abstract}
In this paper, we present some results concerning the existence and the attractivity of solutions for some functional integral equations of Hadamard fractional order. We use an extension of the Burton-Kirk fixed point theorem in Fréchet spaces.
\end{abstract}

Keywords: functional integral equation, Hadamard integral of fractional order, solution, attractivity, Fréchet space, fixed point

MSC: Primary 26A33; Secondary 45G05, 45M10

\section{Introduction}

Fractional integral equations have recently been applied in various areas of engineering, science, finance, applied mathematics, and bio-engineering as well as many other areas. However, many researchers remain unaware of this field. There has been a significant development in ordinary and partial fractional differential and integral equations in recent years; see the monographs of Abbas et al. [1, 2], Baleanu et al. [3], Kilbas et al. [4], Miller and Ross [5], Lakshmikantham et al. [6], Samko et al. [7]. In[8], Butzer et al. investigate properties of the Hadamard fractional integral and derivative. In [9], they obtained the Mellin transforms of the Hadamard fractional integral and differential operators. In [10], Pooseh et al. obtained expansion formulas of the Hadamard operators in terms of integer order derivatives. Many other interesting properties of those operators and others are summarized in [7] and the references therein. Recent interesting existence results on Hadamard fractional differential equations are given in [11-16].

Recently some interesting results on the attractivity of the solutions of some classes of integral equations have been obtained by Abbas et al. [17-20], Banaś et al. [21-24], Darwish et al. [25], Pachpatte [26, 27] and the references therein. This paper deals with the existence and the attractivity of solutions of the following Hadamard fractional integral equation of the form

$$
\begin{aligned}
u(t, x)= & \mu(t, x)+f\left(t, x,{ }^{H} I_{\sigma}^{r} u(t, x), u(t, x)\right)+\frac{1}{\Gamma\left(r_{1}\right) \Gamma\left(r_{2}\right)} \int_{1}^{t} \int_{1}^{x}\left(\log \frac{t}{s}\right)^{r_{1}-1}\left(\log \frac{x}{y}\right)^{r_{2}-1} \\
& \times g(t, x, s, y, u(s, y)) \frac{d y d s}{s y} ;(t, x) \in J:=[1,+\infty) \times[1, b],
\end{aligned}
$$

\footnotetext{
Saïd Abbas: Laboratory of Mathematics, Geometry, Analysis, Control and Applications, Tahar Moulay University of Saïda, P.O. Box 138, EN-Nasr, 20000 Saïda, Algeria, E-mail: said.abbas@univ-saida.dz, abbasmsaid@yahoo.fr

^Corresponding Author: Ravi P. Agarwal: Department of Mathematics, Texas A\&M University-Kingsville, Kingsville, 78363, USA, E-mail: agarwal@tamuk.edu

Mouffak Benchohra: Laboratory of Mathematics, Djillali Liabes University of Sidi Bel-Abbès, P.O. Box 89, Sidi Bel-Abbes 22000, Algeria, E-mail: benchohra@univ-sba.dz

Farida Berhoun: Laboratory of Mathematics, Djillali Liabes University of Sidi Bel-Abbès, P.O. Box 89, Sidi Bel-Abbes 22000, Algeria, E-mail: berhoun22@yahoo.fr
} 
where $b>1, r=\left(r_{1}, r_{2}\right), r_{1}, r_{2} \in(0, \infty),{ }^{H} I_{\sigma}^{r}$ is the Hadamard integral of order $r, \mu: J \rightarrow \mathbb{R}, f: J \times \mathbb{R} \times \mathbb{R} \rightarrow$ $\mathbb{R}, g: J^{\prime} \times \mathbb{R} \rightarrow \mathbb{R}$ are given continuous functions, $J^{\prime}=\left\{(t, x, s, y) \in J^{2}: s \leq t, y \leq x\right\}$ and $\Gamma(\cdot)$ is the (Euler's) Gamma function defined by

$$
\Gamma(\xi)=\int_{0}^{\infty} t^{\xi-1} e^{-t} d t ; \xi>0 .
$$

Our investigations are considered in Fréchet spaces with an application of Burton-Kirk fixed point theorem for the existence of solutions of the integral equation (1.1), and we prove that all solutions are uniformly globally attractive. To our knowledge, the study of integral equations of Hadamard type have not been considered yet in Fréchet spaces, so the present results constitute to contribution to this study.

\section{Preliminaries}

In this section we introduce notations, definitions, and preliminary facts which are used throughout this paper. By $L^{1}([1,+\infty) \times[1, b])$; we denote the space of Lebesgue-integrable functions $u:[1,+\infty) \times[1, b] \rightarrow \mathbb{R}$ with the norm

$$
\|u\|_{1}=\int_{1}^{\infty} \int_{1}^{b}|u(t, x)| d x d t
$$

By $C:=C(J)$ we denote the space of continuous functions from $J$ into $\mathbb{R}$.

Definition 1. $[4,28]$ The Hadamard fractional integral of order $q>0$ for a function $g \in L^{1}([1, a], \mathbb{R})$, is defined as

$$
\left({ }^{H} I_{1}^{q} g\right)(x)=\frac{1}{\Gamma(q)} \int_{1}^{x}\left(\log \frac{x}{s}\right)^{q-1} \frac{g(s)}{s} d s
$$

Example 2. Let $\beta>0$ and let $w:[1, e] \rightarrow \mathbb{R}$ be the function defined by $w(x)=(\log x)^{\beta-1}$. Then the Hadamard fractional integral of order $q>0$ for $w$ is given by

$$
\left({ }^{H} I_{1}^{q} w\right)(x)=\frac{\Gamma(\beta)}{\Gamma(\beta+q)}(\log x)^{\beta+q-1} .
$$

Definition 3. Let $r_{1}, r_{2} \geq 0, \sigma=(1,1)$ and $r=\left(r_{1}, r_{2}\right)$. For $w \in L^{1}(J, \mathbb{R})$, define the Hadamard partial fractional integral of order $r$ by the expression

$$
\left({ }^{H} I_{\sigma}^{r} w\right)(x, y)=\frac{1}{\Gamma\left(r_{1}\right) \Gamma\left(r_{2}\right)} \int_{1}^{x} \int_{1}^{y}\left(\log \frac{x}{s}\right)^{r_{1}-1}\left(\log \frac{y}{t}\right)^{r_{2}-1} \frac{w(s, t)}{s t} d t d s .
$$

Let $X$ be a Fréchet space with a family of semi-norms $\left\{\|\cdot\|_{n}\right\}_{n \in \mathbb{N}^{*}}$. We assume that the family of semi-norms $\left\{\|\cdot\|_{n}\right\}$ verify :

$$
\|x\|_{1} \leq\|x\|_{2} \leq\|x\|_{3} \leq \ldots \text { for every } x \in X .
$$

Let $Y \subset X$, then we say that $Y$ is bounded if for every $n \in \mathbb{N}$, there exists $\bar{M}_{n}>0$ such that

$$
\|y\|_{n} \leq \bar{M}_{n} \text { for all } y \in Y .
$$

To $X$ we associate a sequence of Banach spaces $\left\{\left(X^{n},\|\cdot\|_{n}\right)\right\}$ as follows: First, for every $n \in \mathbb{N}$, we consider the equivalence relation $\sim_{n}$ defined as $x \sim_{n} y$ if and only if $\|x-y\|_{n}=0$ for $x, y \in X$. We denote by $X^{n}=$ $\left(\left.X\right|_{\sim_{n}},\|\cdot\|_{n}\right)$ the quotient space, the completion of $X^{n}$ with respect to $\|\cdot\|_{n}$. To every $Y \subset X$, we associate a sequence $\left\{Y^{n}\right\}$ of subsets $Y^{n} \subset X^{n}$ as follows. For every $x \in X$, we denote by $[x]_{n}$ the equivalence class of $x$ of 
subset $X^{n}$ and we define $Y^{n}=\left\{[x]_{n}: x \in Y\right\}$. We denote by $\overline{Y^{n}}$, int $t_{n}\left(Y^{n}\right)$ and $\partial_{n} Y^{n}$, respectively, the closure, the interior and the boundary of $Y^{n}$ with respect to $\|\cdot\|_{n}$ in $X^{n}$. For more information about this subject see [29].

Definition 4. Let $X$ be a Fréchet space. A function $N: X \longrightarrow X$ is said to be a contraction if for each $n \in \mathbb{N} \backslash\{0\}$ there exists $k_{n} \in[0,1)$ such that

$$
\|N(u)-N(v)\|_{n} \leq k_{n}\|u-v\|_{n} \text { for all } u, v \in X .
$$

We need the following extension of the Burton-Kirk fixed point theorem in the case of a Fréchet space.

Theorem 5. [30] Let $\left(X,\|\cdot\|_{n}\right)$ be a Fréchet space and let $A, B: X \rightarrow X$ be two operators such that

(a) A is a compact operator;

(b) $B$ is a contraction operator with respect to a family of seminorms $\left\{\|\cdot\|_{n}\right\}$;

(c) the set $\left\{x \in X: x=\lambda A(x)+\lambda B\left(\frac{x}{\lambda}\right), \lambda \in(0,1)\right\}$ is bounded.

Then the operator equation $A(u)+B(u)=u$ has a solution in $X$.

Let $\emptyset \neq \Omega \subset C$, and let $G: \Omega \rightarrow \Omega$, and consider the solutions of the equation

$$
(G u)(t, x)=u(t, x) .
$$

Now we introduce the concept of attractivity of solutions for the equation (2.1).

Definition 6. [21] Solutions of the equation (2.1) are locally attractive if there exists a ball $B\left(u_{0}, \eta\right)$ in the space $C$ such that, for arbitrary solutions $v=v(t, x)$ and $w=w(t, x)$ of equation (2.1) belonging to $B\left(u_{0}, \eta\right) \cap \Omega$, we have that, for each $x \in[1, b]$,

$$
\lim _{t \rightarrow \infty}(v(t, x)-w(t, x))=0 .
$$

When the limit (2.2) is uniform with respect to $B\left(u_{0}, \eta\right) \cap \Omega$, solutions of equation (2.1) are said to be uniformly locally attractive (or equivalently that solutions of (2.1) are locally asymptotically stable).

Definition 7. [21] The solution $v=v(t, x)$ of equation (2.1) is said to be globally attractive if (2.2) holds for each solution $w=w(t, x)$ of (2.1). If condition (2.2) is satisfied uniformly with respect to the set $\Omega$, solutions of equation (2.1) are said to be globally asymptotically stable (or uniformly globally attractive).

\section{Existence and attractivity results}

Let us start by defining what we mean by a solution of of the equation (1.1).

Definition 8. A function $u \in C(J)$ is said to be a solution of (1.1) if $u$ satisfies equation (1.1) on $J$.

For each $p \in \mathbb{N} \backslash\{0,1\}$, consider following set, $C_{p}=C([1, p] \times[1, b])$. We define in $C(J)$ the semi-norm by

$$
\|u\|_{p}=\sup _{(t, x) \in[1, p] \times[1, b]}\|u(t, x)\| .
$$

Then $C$ is a Fréchet space with the family of semi-norms $\left\{\|u\|_{p}\right\}$.

Now, we are concerned with the existence and the uniform global attractivity of solutions of the integral equation (1.1). The following hypotheses will be used in the sequel. 
$\left(H_{1}\right)$ There exist continuous functions $l, k: J \rightarrow \mathbb{R}_{+}$, with

$$
\lim _{t \rightarrow \infty} l(t, x)=\lim _{t \rightarrow \infty} k(t, x)=0, \text { for } x \in[1, b],
$$

such that

$$
\left|f\left(t, x, u_{1}, v_{1}\right)-f\left(t, x, u_{2}, v_{2}\right)\right| \leq \frac{l(t, x)\left|u_{1}-u_{2}\right|+k(t, x)\left|v_{1}-v_{2}\right|}{1+\left|u_{1}-u_{2}\right|+\left|v_{1}-v_{2}\right|},
$$

for each $(t, x) \in J$ and each $u_{1}, u_{2}, v_{1}, v_{2} \in \mathbb{R}$.

$\left(H_{2}\right)$ There exist continuous functions $P, Q, \varphi: J^{\prime} \rightarrow \mathbb{R}_{+}$and a nondecreasing function $\psi:[0, \infty) \rightarrow(0, \infty)$ such that

$$
|g(t, x, s, y, u)| \leq \frac{P(t, x, s, y)+Q(t, x, s, y)|u|}{1+|u|} ;
$$

for $(t, x, s, y) \in J^{\prime}, u \in \mathbb{R}$, and

$$
\left|g\left(t_{1}, x_{1}, s, y, u\right)-g\left(t_{2}, x_{2}, s, y, u\right)\right| \leq \varphi(s, y)\left(\left|t_{1}-t_{2}\right|+\left|x_{1}-x_{2}\right|\right) \psi(|u|)
$$

for $\left(t_{1}, x_{1}\right),\left(t_{2}, x_{2}\right),(s, y) \in J$ and $u \in \mathbb{R}$. Moreover, assume that

$$
\lim _{t \rightarrow \infty} \int_{1}^{t}\left|\log \frac{t}{s}\right|^{r_{1}-1} \frac{P(t, x, s, y)}{s} d s=\lim _{t \rightarrow \infty} \int_{1}^{t}\left|\log \frac{t}{s}\right|^{r_{1}-1} \frac{Q(t, x, s, y)}{s} d s=0,
$$

for each $(x, y) \in J$ with $y \leq x$.

Theorem 9. Assume that the hypotheses $\left(H_{1}\right)$ and $\left(H_{2}\right)$ hold. If

$$
k_{p}+\frac{l_{p}(\log p)^{r_{1}}(\log b)^{r_{2}}}{\Gamma\left(1+r_{1}\right) \Gamma\left(1+r_{2}\right)}<1,
$$

where

$$
k_{p}=\sup _{(t, x) \in[1, p] \times[1, b]} k(t, x), l_{p}=\sup _{(t, x) \in[1, p] \times[1, b]} l(t, x) ; p \in \mathbb{N} \backslash\{0,1\},
$$

then the Hadamard integral equation (1.1) has at least one solution in the space C. Moreover, solutions of equation (1.1) are uniformly globally attractive.

Proof. Let us define the operators $A, B: C \rightarrow C$ by

$$
\begin{gathered}
(A u)(t, x)=\int_{1}^{t} \int_{1}^{x}\left(\log \frac{t}{s}\right)^{r_{1}-1}\left(\log \frac{x}{y}\right)^{r_{2}-1} \frac{g(t, x, s, y, u(s, y))}{s y \Gamma\left(r_{1}\right) \Gamma\left(r_{2}\right)} d y d s,(t, x) \in J, \\
(B u)(t, x)=\mu(t, x)+f\left(t, x,{ }^{H} I_{\sigma}^{r} u(t, x), u(t, x)\right),(t, x) \in J .
\end{gathered}
$$

We shall show that the operators $A$ and $B$ satisfy all of the conditions of Theorem 5 . The proof will be given in several steps.

Step 1: $A$ is compact.

To this aim, we must prove that $A$ is continuous and it transforms every bounded set into a relatively compact set. Recall that $M \subset C$ is bounded if and only if,

$$
\forall p \in \mathbb{N} \backslash\{0,1\}, \exists \ell_{p}>0, \text { such that } \forall u \in M,\|u\|_{p} \leq \ell_{p} .
$$

Further, we say that $M=\{u(t, x) ;(t, x)) \in J\} \subset C$ is relatively compact if and only if, for each $p \in \mathbb{N} \backslash\{0,1\}$, the family $\left\{\left.u(t, x)\right|_{(t, x)] \in[1, p] \times[1, b]}\right\}$ is equicontinuous and uniformly bounded on $[1, p] \times[1, b]$. The proof will be given in several claims. 
Claim 1: $A$ is continuous.

Let $\left\{u_{n}\right\}_{n \in \mathbb{N} \backslash\{0,1\}}$ be a sequence in $M$ such that $u_{n} \rightarrow u$ in $M$. Then, for each $(t, x) \in J$, we have

$$
\begin{aligned}
\left|\left(A u_{n}\right)(t, x)-(A u)(t, x)\right| \leq & \frac{1}{\Gamma\left(r_{1}\right) \Gamma\left(r_{2}\right)} \int_{1}^{t} \int_{1}^{x}\left|\log \frac{t}{s}\right|^{r_{1}-1}\left|\log \frac{x}{y}\right|^{r_{2}-1} \\
& \times\left|g\left(t, x, s, y, u_{n}(s, y)\right)-g(t, x, s, y, u(s, y))\right| d y d s \\
\leq & \frac{1}{\Gamma\left(r_{1}\right) \Gamma\left(r_{2}\right)} \int_{1}^{t} \int_{1}^{x}\left|\log \frac{t}{s}\right|^{r_{1}-1}\left|\log \frac{x}{y}\right|^{r_{2}-1} \\
& \times\left|g\left(t, x, s, y, u_{n}(s, y)\right)-g(t, x, s, y, u(s, y))\right| d y d s .
\end{aligned}
$$

If $(t, x) \in[1, p] \times[1, b]$ with $p \in \mathbb{N} \backslash\{0,1\}$ then, since $u_{n} \rightarrow u$ as $n \rightarrow \infty$ and $g$ is continuous, (3.6) gives

$$
\left\|A\left(u_{n}\right)-A(u)\right\|_{p} \rightarrow 0 \quad \text { as } n \rightarrow \infty .
$$

Claim 2: A maps bounded sets into bounded sets in $C$.

Let $M$ be the bounded set in $C$ as in Claim 1. Then, for each $p \in \mathbb{N} \backslash\{0,1\}$, there exists $\ell_{p}>0$, such that for all $u \in C$ we have $\|u\|_{p} \leq \ell_{p}$.

For an arbitrary fixed $(t, x) \in[1, p] \times[1, b]$ we have

$$
\begin{aligned}
|(A u)(t, x)| & \leq \frac{1}{\Gamma\left(r_{1}\right) \Gamma\left(r_{2}\right)} \int_{1}^{t} \int_{1}^{x}\left|\log \frac{t}{s}\right|^{r_{1}-1}\left|\log \frac{x}{y}\right|^{r_{2}-1}|g(t, x, s, y, u(s, y))| d y d s \\
& \leq \frac{1}{\Gamma\left(r_{1}\right) \Gamma\left(r_{2}\right)} \int_{1}^{t} \int_{1}^{x}\left|\log \frac{t}{s}\right|^{r_{1}-1}\left|\log \frac{x}{y}\right|^{r_{2}-1} \frac{P(t, x, s, y)+Q(t, x, s, y)|u(s, y)|}{1+|u(s, y)|} d y d s .
\end{aligned}
$$

Thus,

$$
\begin{aligned}
|(A u)(t, x)| & \leq \frac{1}{\Gamma\left(r_{1}\right) \Gamma\left(r_{2}\right)} \int_{1}^{t} \int_{1}^{x}\left|\log \frac{t}{s}\right|^{r_{1}-1}\left|\log \frac{x}{y}\right|^{r_{2}-1}(P(t, x, s, y)+Q(t, x, s, y)) d y d s \\
& \leq P_{p}+Q_{p},
\end{aligned}
$$

where

$$
P_{p}=\sup _{(t, x) \in[1, p] \times[1, b]} \int_{1}^{t} \int_{1}^{x}\left|\log \frac{t}{s}\right|^{r_{1}-1}\left|\log \frac{x}{y}\right|^{r_{2}-1} \frac{P(t, x, s, y)}{\Gamma\left(r_{1}\right) \Gamma\left(r_{2}\right)} d y d s
$$

and

$$
Q_{p}=\sup _{(t, x) \in[1, p] \times[1, b]} \int_{1}^{t} \int_{1}^{x}\left|\log \frac{t}{s}\right|^{r_{1}-1}\left|\log \frac{x}{y}\right|^{r_{2}-1} \frac{Q(t, x, s, y)}{\Gamma\left(r_{1}\right) \Gamma\left(r_{2}\right)} d y d s
$$

Thus

$$
\|A(u)\|_{p} \leq P_{p}+Q_{p}:=\ell_{p}^{\prime}
$$


Claim 3: A maps bounded sets into equicontinuous sets in $C$.

Let $\left(t_{1}, x_{1}\right),\left(t_{2}, x_{2}\right) \in[1, p] \times[1, b], t_{1}<t_{2}, x_{1}<x_{2}$ and let $u \in M$. We have

$$
\begin{aligned}
\left|(A u)\left(t_{2}, x_{2}\right)-(A u)\left(t_{1}, x_{1}\right)\right| & \leq\left.\frac{1}{\Gamma\left(r_{1}\right) \Gamma\left(r_{2}\right)}\left|\int_{1}^{t_{2}} \int_{1}^{x_{2}}\right| \log \frac{t_{2}}{s}\right|^{r_{1}-1}\left|\log \frac{x_{2}}{y}\right|^{r_{2}-1} \\
& \times\left[g\left(t_{2}, x_{2}, s, y, u(s, y)\right)-g\left(t_{1}, x_{1}, s, y, u(s, y)\right)\right] d y d s \mid \\
& +\left.\frac{1}{\Gamma\left(r_{1}\right) \Gamma\left(r_{2}\right)}\left|\int_{1}^{t_{2}} \int_{1}^{x_{2}}\right| \log \frac{t_{2}}{s}\right|^{r_{1}-1}\left|\log \frac{x_{2}}{y}\right|^{r_{2}-1} g\left(t_{1}, x_{1}, s, y, u(s, y)\right) d y d s \\
& -\int_{1}^{t_{2}} \int_{1}^{x_{2}}\left|\log \frac{t_{1}}{s}\right|^{r_{1}-1}\left|\log \frac{x_{1}}{y}\right|^{r_{2}-1} g\left(t_{1}, x_{1}, s, y, u(s, y)\right) d y d s \mid \\
& +\left.\frac{1}{\Gamma\left(r_{1}\right) \Gamma\left(r_{2}\right)}\left|\int_{1}^{t_{2}} \int_{1}^{x_{2}}\right| \log \frac{t_{1}}{s}\right|^{r_{1}-1}\left|\log \frac{x_{1}}{y}\right|^{r_{2}-1} g\left(t_{1}, x_{1}, s, y, u(s, y)\right) d y d s \\
& -\int_{1}^{t_{1}} \int_{1}^{x_{1}}\left|\log \frac{t_{1}}{s}\right|^{r_{1}-1}\left|\log \frac{x_{1}}{y}\right|^{r_{2}-1} g\left(t_{1}, x_{1}, s, y, u(s, y)\right) d y d s \mid .
\end{aligned}
$$

Then, we get

$$
\begin{aligned}
\left|(A u)\left(t_{2}, x_{2}\right)-(A u)\left(t_{1}, x_{1}\right)\right| & \leq \frac{1}{\Gamma\left(r_{1}\right) \Gamma\left(r_{2}\right)} \int_{1}^{t_{2}} \int_{1}^{x_{2}}\left|\log \frac{t_{2}}{s}\right|^{r_{1}-1}\left|\log \frac{x_{2}}{y}\right|^{r_{2}-1} \\
& \times\left|g\left(t_{2}, x_{2}, s, y, u(s, y)\right)-g\left(t_{1}, x_{1}, s, y, u(s, y)\right)\right| d y d s \\
& +\frac{1}{\Gamma\left(r_{1}\right) \Gamma\left(r_{2}\right)} \int_{1}^{t_{1}} \int_{1}^{x_{1}} \mid\left(\log \frac{t_{2}}{s}\right)^{r_{1}-1}\left(\log \frac{x_{2}}{y}\right)^{r_{2}-1} \\
& -\left(\log \frac{t_{1}}{s}\right)^{r_{1}-1}\left(\log \frac{x_{1}}{y}\right)^{r_{2}-1}|\times| g\left(t_{1}, x_{1}, s, y, u(s, y)\right) \mid d y d s \\
& +\frac{1}{\Gamma\left(r_{1}\right) \Gamma\left(r_{2}\right)} \int_{1}^{t_{1}} \int_{x_{1}}^{x_{2}}\left|\log \frac{t_{2}}{s}\right|^{t_{1}-1}\left|\log \frac{x_{2}}{y}\right|^{t_{2}-1}\left|g\left(t_{1}, x_{1}, s, y, u(s, y)\right)\right| d y d s \\
& +\frac{1}{\Gamma\left(r_{1}\right) \Gamma\left(r_{2}\right)} \int_{t_{1}}^{x_{1}} \int_{1}^{x_{1}}\left|\log \frac{t_{2}}{s}\right|^{t_{1}-1}\left|\log \frac{x_{2}}{y}\right|^{t_{2}-1}\left|g\left(t_{1}, x_{1}, s, y, u(s, y)\right)\right| d y d s \\
& +\frac{1}{\Gamma\left(r_{1}\right) \Gamma\left(r_{2}\right)} \int_{t_{1}}^{x_{2}} \int_{x_{1}}^{r_{1}}\left|\log \frac{t_{2}}{s}\right|^{r_{1}-1}\left|\log \frac{x_{2}}{y}\right|^{r_{2}-1}\left|g\left(t_{1}, x_{1}, s, y, u(s, y)\right)\right| d y d s .
\end{aligned}
$$


Thus, we obtain

$$
\begin{aligned}
\left|(A u)\left(t_{2}, x_{2}\right)-(A u)\left(t_{1}, x_{1}\right)\right| & \leq \frac{1}{\Gamma\left(r_{1}\right) \Gamma\left(r_{2}\right)} \int_{1}^{t_{2}} \int_{1}^{x_{2}}\left|\log \frac{t_{2}}{s}\right|^{r_{1}-1}\left|\log \frac{x_{2}}{y}\right|^{r_{2}-1} \\
& \times \varphi(s, y)\left(\left|t_{1}-t_{2}\right|+\left|x_{1}-x_{2}\right|\right) \psi\left(\ell_{p}\right) d y d s \\
& +\frac{1}{\Gamma\left(r_{1}\right) \Gamma\left(r_{2}\right)} \int_{1}^{t_{1}} \int_{1}^{x_{1}}\left|\left(\log \frac{t_{2}}{s}\right)^{r_{1}-1}\left(\log \frac{x_{2}}{y}\right)^{r_{2}-1}-\left(\log \frac{t_{1}}{s}\right)^{r_{1}-1}\left(\log \frac{x_{1}}{y}\right)^{r_{2}-1}\right| \\
& \times\left(P\left(t_{1}, x_{1}, s, y\right)+Q\left(t_{1}, x_{1}, s, y\right)\right) d y d s \\
& +\frac{1}{\Gamma\left(r_{1}\right) \Gamma\left(r_{2}\right)} \int_{t_{2}}^{t_{2}} \int_{1}^{x_{2}}\left|\log \frac{t_{2}}{s}\right|^{r_{1}-1}\left|\log \frac{x_{2}}{y}\right|^{r_{2}-1}\left(P\left(t_{1}, x_{1}, s, y\right)+Q\left(t_{1}, x_{1}, s, y\right)\right) d y d s \\
& +\frac{1}{\Gamma\left(r_{1}\right) \Gamma\left(r_{2}\right)} \int_{1}^{t_{1}} \int_{x_{1}}^{x_{2}}\left|\log \frac{t_{2}}{s}\right|^{r_{1}-1}\left|\log \frac{x_{2}}{y}\right|^{r_{2}-1}\left(P\left(t_{1}, x_{1}, s, y\right)+Q\left(t_{1}, x_{1}, s, y\right)\right) d y d s \\
& +\frac{1}{\Gamma\left(r_{1}\right) \Gamma\left(r_{2}\right)} \int_{t_{1}}^{r_{2}} \int_{x_{1}}^{x_{2}}\left|\log \frac{t_{2}}{s}\right|^{r_{1}-1}\left|\log \frac{x_{2}}{y}\right|^{r_{2}-1}\left(P\left(t_{1}, x_{1}, s, y\right)+Q\left(t_{1}, x_{1}, s, y\right)\right) d y d s .
\end{aligned}
$$

From the continuity of the functions $P, Q, \varphi$, and as $t_{1} \longrightarrow t_{2}$ and $x_{1} \longrightarrow x_{2}$, the right-hand side of the above inequality tends to zero. As a consequence of claims 1-3, and from the Arzelá-Ascoli theorem, we can conclude that $A$ is continuous and compact.

Step 2: $B$ is a contraction.

Consider $v, w \in C$. Then, by $\left(H_{1}\right)$, for any $p \in \mathbb{N} \backslash\{0,1\}$ and each $(t, x) \in[1, p] \times[1, b]$, we have

$$
\begin{aligned}
|(B v)(t, x)-(B w)(t, x)| & \leq\left. l(t, x)\right|^{H} I_{\sigma}^{r}(v-w)(t, x)|+k(t, x)|(v-w)(t, x) \mid \\
& \leq\left(k(t, x)+\frac{l(t, x)(\log p)^{r_{1}}(\log b)^{r_{2}}}{\Gamma\left(1+r_{1}\right) \Gamma\left(1+r_{2}\right)}\right)|v-w| .
\end{aligned}
$$

Thus,

$$
\|\left(B(v)-B(w)\left\|_{p} \leq\left(k_{p}+\frac{l_{p}(\log p)^{r_{1}}(\log b)^{r_{2}}}{\Gamma\left(1+r_{1}\right) \Gamma\left(1+r_{2}\right)}\right)\right\| v-w \|_{p} .\right.
$$

By (3.3), we conclude that $B$ is a contraction.

Step 3: The set $\mathcal{E}:=\left\{u \in C(J): u=\lambda A(u)+\lambda B\left(\frac{u}{\lambda}\right), \lambda \in(0,1)\right\}$ is bounded.

Let $u \in C(J)$, such that $u=\lambda A(u)+\lambda B\left(\frac{u}{\lambda}\right)$ for some $\lambda \in(0,1)$. Then, for any $p \in \mathbb{N} \backslash\{0,1\}$ and each $(t, x) \in[1, p] \times[1, b]$, we have

$$
\begin{aligned}
|u(t, x)| & \leq \lambda|A(u)|+\lambda\left|B\left(\frac{u}{\lambda}\right)\right| \\
& \leq|\mu(t, x)|+|f(t, x, 0,0)|+k(t, x)+l(t, x) \\
& +\frac{1}{\Gamma\left(r_{1}\right) \Gamma\left(r_{2}\right)} \int_{1}^{t} \int_{1}^{x}\left|\log \frac{t}{s}\right|^{r_{1}-1}\left|\log \frac{x}{y}\right|^{r_{2}-1} \frac{P(t, x, s, y)+Q(t, x, s, y)}{s y} d y d s \\
& \leq \mu_{p}+f_{p}+k_{p}+l_{p}+P_{p}+Q_{p},
\end{aligned}
$$

where

$$
\mu_{p}=\sup _{(t, x) \in[1, p] \times[1, b]} \mu(t, x), f_{p}=\sup _{(t, x) \in[1, p] \times[1, b]}|f(t, x, 0,0)| .
$$

Thus,

$$
\|u\|_{p} \leq \mu_{p}+f_{p}+k_{p}+l_{p}+P_{p}+Q_{p}=: \ell_{p}^{\star}
$$


Hence, the set $\mathcal{E}$ is bounded.

As a consequence of steps 1-3 and Theorem 5 we deduce that $N$ has a fixed point $u$ which is a solution of the integral equation (1.1).

Step 4: The uniform global attractivity of solutions of the integral equation (1.1). Let $u$ and $v$ be any two solutions of (1.1), then for each $(t, x) \in J$ we have

$$
\begin{aligned}
|u(t, x)-v(t, x)| \leq & \left|f\left(t, x,{ }^{H} I_{\sigma}^{r} u(t, x), u(t, x)\right)-f\left(t, x,{ }^{H} I_{\sigma}^{r} v(t, x), v(t, x)\right)\right| \\
& +\frac{1}{\Gamma\left(r_{1}\right) \Gamma\left(r_{2}\right)} \int_{1}^{t} \int_{1}^{x}\left|\log \frac{t}{s}\right|^{r_{1}-1}\left|\log \frac{x}{y}\right|^{r_{2}-1} \\
& \times|g(t, x, s, y, u(s, y))-g(t, x, s, y, v(s, y))| \frac{d y d s}{s y} \\
\leq & k(t, x)+l(t, x)+\frac{2}{\Gamma\left(r_{1}\right) \Gamma\left(r_{2}\right)} \int_{1}^{t} \int_{1}^{x}\left|\log \frac{t}{s}\right|^{r_{1}-1}\left|\log \frac{x}{y}\right|^{r_{2}-1} \\
& \times \frac{P(t, x, s, y)+Q(t, x, s, y)}{s y} d y d s \\
\leq & k(t, x)+l(t, x)+\frac{2}{\Gamma\left(r_{1}\right) \Gamma\left(r_{2}\right)} \int_{1}^{x}\left|\log \frac{x}{y}\right|^{r_{2}-1} \\
& \times\left(\int_{1}^{t}\left|\log \frac{t}{s}\right|^{r_{1}-1}(P(t, x, s, y)+Q(t, x, s, y)) \frac{d s}{s}\right) \frac{d y}{y} .
\end{aligned}
$$

Thus, by (3.1) and (3.2), we deduce that

$$
\lim _{t \rightarrow \infty}(u(t, x)-v(t, x))=0
$$

Consequently, all solutions of the integral equation (1.1) are uniformly globally attractive.

\section{An example}

Consider the following Hadamard fractional order integral equation

$$
\begin{aligned}
& u(t, x)=\frac{x e^{3-2 t}}{1+t+x^{2}}+\frac{x e^{-t-2}}{c_{p}\left(1+\left.e^{-2 p}\right|^{H} I_{\sigma}^{r} u(t, x)\left|+e^{-p}\right| u(t, x) \mid\right)} \\
& +\int_{1}^{t} \int_{1}^{x}\left(\log \frac{t}{s}\right)^{r_{1}-1}\left(\log \frac{x}{y}\right)^{r_{2}-1} \frac{g(t, x, s, y, u(s, y))}{\Gamma\left(r_{1}\right) \Gamma\left(r_{2}\right)} d y d s,
\end{aligned}
$$

for $(t, x) \in[1,+\infty) \times[1, e]$, where $c_{p}=e^{-p}+\frac{e^{-2 p} p^{r_{1}}}{\Gamma\left(1+r_{1}\right) \Gamma\left(1+r_{2}\right)}, p \in \mathbb{N} \backslash\{0,1\}, r=\left(r_{1}, r_{2}\right) \in(0, \infty) \times(0, \infty)$ and

$$
g(t, x, s, y, u)=\frac{x s^{\frac{-3}{4}}(1+|u|) \sin \sqrt{t} \sin s}{\left(1+y^{2}+t^{2}\right)(1+|u|)}, \text { for }(t, x, s, y) \in J^{\prime}, \text { and } u \in \mathbb{R} \text {, }
$$

and

$$
J^{\prime}=\{(t, x, s, y): 1 \leq s \leq t \text { and } 1 \leq x \leq y \leq e\}
$$

Set

$$
\mu(t, x)=\frac{x e^{3-2 t}}{1+t+x^{2}}, f(t, x, u, v)=\frac{x e^{-t-2}}{c_{p}\left(1+e^{-2 p}|u|+e^{-p}|v|\right)} .
$$


The function $f$ is continuous and satisfies the assumption $\left(H_{1}\right)$, with

$$
k(t, x)=\frac{x e^{-t-2-p}}{c_{p}}, l(t, x)=\frac{x e^{-t-2-2 p}}{c_{p}}, k_{p}=\frac{e^{-2-p}}{c_{p}} \text { and } l_{p}=\frac{e^{-2-2 p}}{c_{p}} .
$$

Also, the function $g$ is continuous and satisfies the assumption $\left(H_{2}\right)$, with

$$
P(t, x, s, y)=Q(t, x, s, y)=\frac{x s^{\frac{-3}{4}} \sin \sqrt{t} \sin s}{1+y^{2}+t^{2}}, \text { where }(t, x, s, y) \in J^{\prime} .
$$

Then,

$$
\begin{aligned}
\left.\left|\int_{1}^{t}\right| \log \frac{t}{s}\right|^{r_{1}-1} \frac{P(t, x, s, y)}{s} d s \mid & \leq \int_{1}^{t}\left|\log \frac{t}{s}\right|^{r_{1}-1} x s^{-1}|\sin \sqrt{t} \sin s| d s \\
& \leq\left. x\left|\sin \sqrt{t} \int_{1}^{t}\right| \log \frac{t}{s}\right|^{r_{1}-1} s^{-1} d s \\
& \leq e\left|\frac{(\log t)^{r_{1}}}{\sqrt{t}}\right| \longrightarrow 0 \text { as } t \rightarrow \infty .
\end{aligned}
$$

Finally, we show that condition (3.3) holds with $b=e$. Indeed, for each $p \in \mathbb{N} \backslash\{0,1\}$, we get

$$
k_{p}+\frac{l_{p}(\log p)^{r_{1}}(\log b)^{r_{2}}}{\Gamma\left(1+r_{1}\right) \Gamma\left(1+r_{2}\right)}=\frac{1}{c_{p}}\left(e^{-2-p}+\frac{e^{-2-2 p} p^{r_{1}}}{\Gamma\left(1+r_{1}\right) \Gamma\left(1+r_{2}\right)}\right)=e^{-2}<1 .
$$

Hence by Theorem 9, the equation (4.1) has a solution defined on $[1,+\infty) \times[1, e]$ and all the solutions are uniformly globally attractive.

\section{References}

[1] Abbas S., Benchohra M., N'Guérékata G. M., Topics in Fractional Differential Equations, Developments in Mathematics, 27, Springer, New York, 2012

[2] Abbas S., Benchohra M., N’Guérékata G. M., Advanced Fractional Differential and Integral Equations, Nova Science Publishers, New York, 2015

[3] Baleanu D., Diethelm K., Scalas E., Trujillo J. J., Fractional Calculus Models and Numerical Methods, World Scientific Publishing, New York, 2012

[4] Kilbas A. A., Srivastava H. M., Trujillo J. J., Theory and Applications of Fractional Differential Equations, Elsevier Science B.V., Amsterdam, 2006

[5] Miller K. S., Ross B., An Introduction to the Fractional Calculus and Differential Equations, John Wiley, New York, 1993

[6] Lakshmikantham V., Leela S., Vasundhara J., Theory of Fractional Dynamic Systems, Cambridge Academic Publishers, Cambridge, 2009

[7] Samko S. G., Kilbas A. A., Marichev O. L., Fractional Integrals and Derivatives, Theory and Applications, Gordon and Breach, Yverdon, 1993

[8] Butzer P. L., Kilbas A. A., Trujillo J. J., Fractional calculus in the Mellin setting and Hadamard-type fractional integrals, J. Math. Anal. Appl., 2002, 269, 1-27

[9] Butzer P. L., Kilbas A. A., Trujillo J. J., Mellin transform analysis and integration by parts for Hadamard-type fractional integrals, J. Math. Anal. Appl., 2002, 270, 1-15

[10] Pooseh S., Almeida R., Torres D., Expansion formulas in terms of integer-order derivatives for the Hadamard fractional integral and derivative, Numer. Funct. Anal. Optim., 2012, 33(3), 301-319

[11] Abbas A., Alaidarous E., Benchohra M., Nieto J. J, Existence and stability of solutions for Hadamard-Stieltjes fractional integral equations, Discrete Dyn. Nat. Soc., 2015, Art. ID 317094

[12] Adjabi Y., Jarad F., Baleanu D., Abdeljawad T., On Cauchy problems with Caputo Hadamard fractional derivatives, J. Comput. Anal. Appl., 2016, 21(4), 661-681

[13] Aljoudi S., Ahmad B., Nieto J. J., Alsaedi A., A coupled system of Hadamard type sequential fractional differential equations with coupled strip conditions, Chaos Solitons Fractals, 2016, 91, 39-46

[14] Benchohra M., Bouriah S., Nieto J. J., Existence of periodic solutions for nonlinear implicit Hadamard's fractional differential equations, Rev. R. Acad. Cienc. Exactas, Fís. Nat. Ser. A Math. RACSAM, 2018, 112, 25-35 
[15] Gambo Y. Y., Jarad F., Baleanu D., Abdeljawad T., On Caputo modification of the Hadamard fractional derivatives, Adv. Difference Equ., 2014, 2014:10

[16] Wang G., Pei K., Baleanu D., Explicit iteration to Hadamard fractional integro-differential equations on infinite domain, Adv. Difference Equ., 2016, 2016:299

[17] Abbas S., Benchohra M., Nonlinear quadratic Volterra Riemann-Liouville integral equations of fractional order, Nonlinear Anal. Forum, 2012, 17, 1-9

[18] Abbas S., Benchohra M., Fractional order Riemann-Liouville integral equations with multiple time delay, Appl. Math. ENotes, 2012, 12, 79-87

[19] Abbas S., Benchohra M., Henderson J., On global asymptotic stability of solutions of nonlinear quadratic Volterra integral equations of fractional order, Comm. Appl. Nonlinear Anal., 2012, 19, 79-89

[20] Abbas S., Benchohra M., Vityuk A. N., On fractional order derivatives and Darboux problem for implicit differential equations, Fract. Calc. Appl. Anal., 2012, 15(2), 168-182

[21] Banaś J., Dhage B. C., Global asymptotic stability of solutions of a functional integral equation, Nonlinear Anal., 2008, 69(7), 1945-1952

[22] Banaś J., Rzepka B., On existence and asymptotic stability of solutions of a nonlinear integral equation, J. Math. Anal. Appl., 2003, 284, 165-173

[23] Banaś J., Zając T., Solvability of a functional integral equation of fractional order in the class of functions having limits at infinity, Nonlinear Anal., 2009, 71, 5491-5500

[24] Banaś J., Zając T., A new approach to the theory of functional integral equations of fractional order, J. Math. Anal. Appl., 2011, 375, 375-387

[25] Darwish M. A., Henderson J., O’Regan D., Existence and asymptotic stability of solutions of a perturbed fractional functional integral equations with linear modification of the argument, Bull. Korean Math. Soc., 2011, 48(3), 539-553

[26] Pachpatte B. G., On Volterra-Fredholm integral equation in two variables, Demonstratio Math., 2007, XL(4), 839-852

[27] Pachpatte B. G., On Fredholm type integral equation in two variables, Differ. Equ. Appl., 2009, 1, 27-39

[28] Hadamard J., Essai sur L'étude des Fonctions Données par Leur Développment de Taylor, J. Pure Appl. Math., 1892, 4(8), 101-186

[29] Frigon M., Granas A., Théorèmes d’Existence pour des Inclusions Différentielles sans Convexité, C. R. Acad. Sci. Paris, Ser. I, 1990, 310, 819-822

[30] Avramescu C., Some remarks on a fixed point theorem of Krasnoselskii, Electron. J. Qual. Theory Differ. Equ., 2003, 5, 1-15 